\title{
Highlights from ACC 15 Scientific Sessions: Part 2
}

\author{
Cihangir Kaymaz, Lale Tokgözoğlu'
}

Clinic of Cardiology, Kartal Koşuyolu Yüksek İhtisas Education and Research Hospital; İstanbul-Turkey ${ }^{1}$ Department of Cardiology, Faculty of Medicine, Hacettepe University; Ankara-Turkey

The surgical ablation of atrial fibrillation (AF) during mitral valve surgery versus mitral valve surgery alone. This study was presented by Marc Gillinov, MD, and was simultaneously published online in the New England Journal of Medicine. 260 patients with persistent or long-standing persistent AF who underwent mitral-valve surgery were randomly assigned to either surgical ablation (ablation group) or no ablation (control group) during the mitral-valve operation. Patients in the ablation group underwent further randomization to pulmonary vein isolation or a biatrial maze procedure. The left atrial appendage was closed in all patients. The primary end point was freedom from $\mathrm{AF}$ at both 6 months and 12 months (as assessed by means of 3-day Holter monitoring). The rate of freedom from AF at both 6 and 12 months without subsequent procedures was $63.2 \%$ with the dual procedure compared with $29.4 \%$ after mitral valve surgery alone $(p<0.001)$. There was no difference in the rate of freedom from AF between patients who underwent pulmonaryvein isolation and those who underwent the biatrial maze procedure $(61.0 \%$ and $66.0 \%$, respectively; $\mathrm{p}=0.60)$. One-year mortality was $6.8 \%$ in the ablation group and $8.7 \%$ in the control group hazard ratio (HR) with ablation, $0.76 ; 95 \%$ confidence interval, 0.32 to $1.84 ; \mathrm{p}=0.55$ ). Ablation compared with no ablation increased the risk of permanent pacemaker implantation (21.5 vs. 8.1 per 100 patient-years, $\mathrm{p}=0.01$ ). The major cardiac or cerebrovascular adverse events, overall serious adverse events, or hospital readmissions were similar beween ablation and no ablation arms $(\mathrm{p}>0.05)$. In conclusion, the addition of $A F$ ablation to mitral-valve surgery significantly improved the rate of freedom from $A F$ at 1 year among patients with persistent or longstanding persistent $A F$ with the expense of the increased risk of a permanent pacemaker implantation (1).

AATAC-AF in Heart Failure: Ablation vs. Amiodarone for Treatment of Persistent Atrial Fibrillation in Patients With
Congestive Heart Failure and an Implanted device: Ablation reduced hospitalization rates and mortality compared with amiodarone treatment. The results of this multicenter, randomized trial were presented by Luigi Di Biase, MD. This trial included 203 patients over the age of 18 who had persistent $A F_{\text {, }}$ New York Heart Association class II or III heart failure, a left ventricular ejection fraction of $40 \%$ or less, and a dual chamber implantable cardioverter defibrillator or cardiac resynchronization therapy device,and patients were randomized to be treated with catheter ablation or amiodarone. The main goal of the ablation procedure was pulmonary vein antrum isolation, but additional linear lesions, ablation of complex fractionated electrogram and elimination of non-pulmonary vein triggers were also encouraged. In the ablation arm, a second ablation was allowed in the 3-month blanking period. Two groups had comparable left atrium size, left ventricular ejection fraction, or median AF duration. Patients were followed for 2 years. The primary endpoint was recurrence of $A F$, and long-term procedural success was defined as freedom of $A F$, atrial flutter, or atrial tachycardia of greater than 30 seconds off antiarrhythmic drugs. At 26 months, freedom from recurrence of AF was significantly higher in the ablation arm compared with the amiodarone arm $(70 \%$ vs. $34 \%, p<0.001)$. There was a higher success rate in patients who underwent pulmonary vein isolation in addition to ablation of non-pulmonary vein triggers $(78.8 \%$ vs. $36.4 \%, \mathrm{p}<0.001)$. In the amiodarone arm $10.4 \%$ of the patients discontinued the medication because of the side effects. All-cause mortality and hospitalization rate over 2 years were significantly lower in the ablation group $(18 \%$ vs. $8 \%, p=0.037$ and $57 \%$ vs. $31 \%, p<0.001$, respectively). Freedom from AF recurrence related to significant improvements in left ventricular ejection fraction (improvement of $9.6 \%$ vs. $4.2 \%$, $\mathrm{p}<0.001)$, as well as 6 -minute walking distance and quality of life measures (2).

Address for Correspondence: Dr. Cihangir Kaymaz, Kartal Koşuyolu Yüksek İhtisas Eğitim ve Araştırma Hastanesi Kardiyoloji Kliniği, 34656, Kartal, Istanbul-Türkiye Phone: + 902165001500 Fax: +90 2164596321 E-mail: cihangirkaymaz2002@yahoo.com

Accepted Date: 12.05.2015

(C) Copyright 2015 by Turkish Society of Cardiology - Available online at www.anatoljcardiol.com DOl:10.5152/akd.2015.00107 
ENGAGE AF-TIMI 48 study: Genetic-Based Sensitivity To Warfarin is associated with a higher risk of bleeding within first 90 days of treatment. This study were published simultaneously in the Lancet, March 2015. Of 4833 patients taking warfarin, $61.7 \%$ were classified as normal responder, $35.4 \%$ as sensitive responders, and $2.9 \%$ as highly sensitive responders. A subgroup of patients was included in a prespecified genetic analysis and genotyped for variants in CYP2C9 and VKORC1. Compared with normal responders, sensitive and highly sensitive responders had greater proportions of time over-anticoagulated in the first 90 days of treatment (median $2.2 \%$, IQR 0-20.2; 8.4\%, 0-25.8; and 18.3\%, 0-32.6; $p_{\text {trend }}<0.0001$ ) translated to increased risk of bleeding with warfarin (sensitive responders HR 1.31, 95\% Cl 1.05-1.64, $\mathrm{p}=0.0179$; highly sensitive responders HR 2.66, 95\% Cl 1.69-4.19, $\mathrm{p}<0.0001)$. Genotype added independent information beyond clinical risk scoring. During the first 90 days, when compared with warfarin, treatment with edoxaban reduced bleeding more so in sensitive and highly sensitive responders than in normal responders (higher-dose edoxaban $\mathrm{p}_{\text {interaction }}=0.0066$; lower-dose edoxaban $\mathrm{p}_{\text {interaction }}=0.0036$ ). After 90 days, the reduction in bleeding risk with edoxaban versus warfarin was similarly effective across genotypes. In conclusion, CYP2C9 and VKORC1 genotypes identify patients prone to early bleeding with warfarin and who derive a greater early safety benefit from edoxaban compared with warfarin (3).

LEGACY (Long-Term Effect of Goal Directed Weight Management in an Atrial Fibrillation Cohort: A Long-term Follow-Up Study): Weight loss improves AF symptoms but fluctuation worsens. LEGACY study was presented by Rajeev K. Pathak , and was concurrently published in the Journal of the American College of Cardiology. Three separate weight-loss groups were constructed from 355 participating patients: group one had a weight loss of 10 percent or more, group two had weight loss between 3 and 9 percent, while group three lost 3 percent or less of their initial body weight. Follow-up period was 5 years. Weight loss had a stepwise influence on arrhythmiafree survival, and it was six times greater among those who achieved and maintained more than 10 percent weight-loss over one year. Weight loss also influenced cardiac structural remodeling and improved left atrium volume index, interventricular septum thickness and left ventricular end-diastolic diameter among patients in groups one and two. However, weight fluctuation of more than 5 percent doubled the risk of recurrent arrhythmia. In conclusion, weight loss in obese patients, improved the rate of the freedom from $A F$ while more than 5 percent fluctuation in weight over one year increased the risk of AF recurrence (4).

ERRICA trial: Remote ischemic conditioning prior to onpump CABG in high-risk patients fails to reduce long-term adverse events. Derek J. Hausenloy, M.D. presented ERRICA trial in which 1,612 patients undergoing on-pump CABG at 29 hospitals in the United Kingdom were randomized to receive remote ischemic preconditioning $(n=801)$ or a sham procedure $(\mathrm{n}=811)$ Mean additive EuroSCORE was 6.7, and diabetes was present $25 \%$ of study population. Preconditioning was performed using intermittent arm ischemia via 4 cycles of 5 -minute inflation followed by 5-minute deflation of a standard bloodpressure cuff after anesthesia induction but before surgical incision. Perioperative myocardial injury was measured by the area under the curve of high-sensitivity troponin $T$ at $6,12,24,48$, and 72 hours. Although preconditioning reduced perioperative myocardial injury by about $10 \%(p=0.039)$, there was no difference in the primary endpoint of MACCE (cardiovascular death, $\mathrm{MI}$, stroke, and coronary revascularization) or any of its individual outcomes between the groups at 1 year (5).

Positive results from proprotein convertase subtilisinkexin type 9 (PCSK9) inhibitors; Evolocumab (OSLER-1,0SLER2) (6) and Alirocumab (ODYSSEY LONG TERM) (7) trials

Combined analysis of OSLER-1 and OSLER-2 trials: OpenLabel Study of Long-Term Evaluation against LDL Cholesterol: Evolocumab plus standard therapy versus standard therapy alone, significantly reduced LDL cholesterol levels and incidence of cardiovascular events. Two openlabel, randomized traials (OSLER-1 and OSLER-2) of phase 2 or 3 studies ("parent trials") were designed to evaluate longer-term data on efficacy of Evolocumab, a monoclonal antibody inhibiting PCSK9. These trials enrolled 4465 patients who had completed phase 2 or 3 studies of evolocumab. Regardless of study group assignments in the parent trials, eligible patients were randomly assigned in a 2:1 ratio to receive either evolocumab (140 mg every 2 weeks or $420 \mathrm{mg}$ monthly) plus standard therapy or standard therapy alone. Patients were followed for a median of 11.1 months with assessment of lipid levels, safety, and (as a prespecified exploratory analysis) adjudicated cardiovascular events including death, $\mathrm{MI}$, unstable angina, coronary revascularization, stroke, transient ischemic attack, and heart failure. Data from the two trials were combined. As compared with standard therapy alone, evolocumab reduced the level of low-density lipoprotein (LDL) cholesterol by $61 \%(p<0.001)$. Although neurocognitive events were more frequent with evolocumab, the risk of adverse events, including neurocognitive events, did not vary significantly according to the achieved level of LDL cholesterol. The rate of cardiovascular events at 1 year was reduced from $2.18 \%$ in the standardtherapy group to $0.95 \%$ in the evolocumab group (HRin the evolocumab group, $0.47 ; 95 \% \mathrm{Cl}, 0.28$ to $0.78 ; \mathrm{p}=0.003$ ). In summary, during approximately 1 year of therapy, the use of evolocumab plus standard therapy, as compared with standard therapy alone, significantly reduced LDL cholesterol levels and the incidence of cardiovascular events in a prespecified but exploratory analysis (6). 
ODYSSEY LONG TERM: Long-term Safety and Tolerability of Alirocumab in High Cardiovascular Risk Patients with Hypercholesterolemia Not Adequately Controlled with Their Lipid Modifying Therapy. Alirocumab, when added to statin therapy at the maximum tolerated dose, significantly reduced LDL cholesterol level and rate of cardiovascular events. The efficacy and safety of Alirocumab, a monoclonal antibody that inhibits PCSK9 were tested in this randomized trial involving 2341 patients at high risk for cardiovascular events who had LDL cholesterol levels of $70 \mathrm{mg}$ per deciliter or more and were receiving treatment with statins at the maximum tolerated dose, with or without other lipid-lowering therapy. Patients were randomly assigned in a 2:1 ratio to receive alirocumab $(150 \mathrm{mg})$ or placebo as a 1-mL subcutaneous injection every 2 weeks for 78 weeks. The primary efficacy end point was the $\%$ change in LDL cholesterol level from baseline to week 24. At week 24, the difference between the alirocumab and placebo groups in the mean percentage change from baseline in calculated LDL cholesterol level was $62 \%(p<0.001)$, and this treatment effect remained consistent over a period of 78 weeks. The alirocumab versus placebo related with had higher rates of injection-site reactions myalgia, neurocognitive events and ophthalmologic events. In a post hoc analysis, the rate of major adverse cardiovascular events (death from coronary heart disease, nonfatal $\mathrm{MI}$, fatal or nonfatal ischemic stroke, or unstable angina requiring hospitalization) was lower with alirocumab than with placebo $(1.7 \%$ vs. $3.3 \%$; $\mathrm{HR}, 0.52 ; 95 \% \mathrm{Cl}, 0.31$ to $0.90 ; \mathrm{p}=0.02)$. In conclusion, alirocumab, when added to statin therapy at the maximum tolerated dose, significantly reduced LDL cholesterol levels. Posthoc analysis indicated a reduction in the rate of cardiovascular events with alirocumab. The ODYSSEY LONG TERM and OSLER studies was simultaneously published online on March 15, 2015, at NEJM.org. (7).

In their editorial on two studies, (8) Neil J. Stone, M.D., and Donald M. Lloyd-Jones, M.D. wrote that "The ODYSSEY LONG TERM and OSLER studies whet our appetites for further results that show cardiovascular benefit and documented safety, even at substantially lower LDL cholesterol ranges than achieved before. However, it would be premature to endorse these drugs for widespread use before the ongoing randomized trials, appropriately powered for primary endpoint analysis and safety assessment, are available." "What's new? Both studies provide post hoc analyses showing approximately 50\% reductions in composite cardiovascular events at 12 to 18 months." "The evidence-driven cholesterol guidelines did not endorse the concept that lower LDL cholesterol levels are better at all costs. They emphasized that, while lower is better, it matters how you get there and whether the benefits outweigh the risks for that patient. Much work remains to be done, but PCSK9 inhibitors appear on track to become important arrows in our quiver for targeting reduction of cardiovascular events among higher-risk patients when statins are not enough." Stone and Lloyd-Jones said.
QuickFlex, QuickSite Lead Flaw Found To Be A “Cosmetic Failure." First prospective follow-up of patients given QuickSite and QuickFlex (St Jude Medical) leads for biventricular pacing indicated that conductor wires protruding through the silicone sheath appear to be 'purely a cosmetic failure,' without any evidence of any electrical abnormality in these patients (9).

Advances in treatment allowing individuals with hypertrophic cardiomyopathy to live longer. The people with hypertrophic cardiomyopathy (HCM) " now live longer than they did 10 or 15 years ago" and death rates among individuals with HCM were about the same as among adults in the general population (10).

Energy drinks may raise resting blood pressure among individuals who are caffeine-naïve. Researchers, working with 25 healthy young adults between the ages of 19 and 40 , gave the participants either a can of a commercially available energy drink or a can containing a placebo concoction. The investigators found that a half hour later, all participants who consumed an energy drink underwent a marked elevation in blood pressure compared to those who didn't and the rise was more dramatic in caffeine-naïve participants (11).

A Reverse J-Shaped Association Between Vitamin D And cardiovascular Mortality. This observational cohort study based on data from nearly 250.000 people showed " a reverse J-shaped association between vitamin $D$ and cardiovascular-related mortality - with a stronger association for those with low levels of the hormone." This study indicated that "those with low 25 -hydroxyvitamin D levels-on the order of $12.5 \mathrm{nmoL} / \mathrm{L}$ - had a hazard ratio for cardiovascular disease mortality of $2.0(95 \% \mathrm{Cl}$ 1.8-2.1) compared with members who had levels of $70 \mathrm{nmoL} / \mathrm{L}$ (those with the lowest mortality risk) and men with low 25-OH-D levels were at greater risk (HR 2.5, 95\% $\mathrm{Cl} 2.2-2.9$ ) than were women with low levels (HR 1.7, 95\% Cl: 1.5-1.9)." This study published online in the Journal of Clinical Endocrinology and Metabolism (12).

Anxiety In Teen Years Linked To Higher Risk Of Dying From Heart Attack Four Decades Later. Scott Montgomery, MD, presented that "men who were anxious in their late teens were twice as likely to die from heart attacks four decades later." (13).

Using folic acid supplements added on Enalapril may reduce stroke risk. The study included more than 20.000 adults in China with high blood pressure but no history of stroke or heart attack, and participants were randomly assigned to take a daily pill with folic acid and" enalapril "or a pill with enalapril alone. The findings were published in the Journal of the American Medical Association. Over a median treatment period of 4.5 years, first strokes occurred in $2.7 \%$ of those in the 
enalapril/folic acid group and $3.4 \%$ of those in the enalapril group. Participants taking enalapril/folic acid also had a lower risk of ischemic stroke $(2.2 \%$ vs. $2.8 \%)(14)$.

MAGMA Study: Multi-Analyte, Thrombogenic, and Genetic Markers of Atherosclerosis: Fish-Oil Supplementation (FOS) may reduce overall atherothrombotic risk profile in patients with suspected CAD (15). MAGMA study indicated a significant association between fish-oil supplementation and decreased inflammation, thrombogenicity and lipid markers. The effect was found to be more pronounced for LDL cholesterol, total very-low density lipoprotein (VLDL) cholesterol, and triglycerides.

GRIPHON Study: Selexipag, an orally available, selective IP receptor agonist targeting the prostacyclin pathway, significantly reduced combined morbidity / mortality events in patients with pulmonary arterial hypertension. The GRIPHON trial evaluated the long-term effect of selexipag, on morbidity/ mortality ( $\mathbf{M} / \mathbf{M})$ as well as tolerability and safety in patients with pulmonary arterial hypertension (PAH), and was presented by Vallerie V. McLaughlin, M.D. (16). In this multicenter, doubleblind, placebo-controlled, phase 3 study, 1156 patients were randomly assigned to placebo or selexipag; Stable background PAH therapy with endothelin receptor antagonists (ERA) and/or phosphodiesterase-5 inhibitors (PDE-5i) was allowed. $20 \%$ were PAH therapy naive, $47 \%$ were on monotherapy (ERA or PDE-5i) and $33 \%$ on combination therapy (ERA and PDE-5i) at baseline. Study drug was titrated to an individual highest tolerated dose (from 200 to $1600 \mu \mathrm{g}$ b.i.d.). The primary efficacy endpoint was the time from randomization to first $\mathbf{M} / \mathbf{M}$ event up to the end of treatment, defined as either disease progression [based on $15 \%$ decrease in 6-minute walk, and either worsening of functional class (FC) or need for additional PAH therapy], hospitalization for $\mathrm{PAH}$ worsening, PAH worsening (need for atrial septostomy or lung transplant; initiation of parenteral prostanoids or chronic $\mathrm{O}_{2}$ therapy), or all-cause death. Mean duration of selexipag and placebo treatment was $76.4 \pm 50.45$ and $71.2 \pm 48.32$ weeks, respectively. Selexipag reduced the risk of $\mathbf{M} / \mathbf{M}$ events vs. placebo (log-rank $p<0.0001$ ) by $40 \%$ (HR 0.60; 99\% Cl: 0.46, 0.78). The treatment effect was consistent across age, gender, etiology, baseline FC and background PAH therapy sub-groups. The most frequent adverse events (selexipag $>3 \%$ over placebo) were headache, diarrhea, nausea, jaw pain, myalgias, pain in extremity, flushing and arthralgia, consistent with prostacyclin effects. In conclusion, Selexipag demonstrated a significant effect on the time to first $\mathbf{M} / \mathbf{M}$ event in PAH patients irrespective of background treatment with ERA, PDE-5i, or both, and had an acceptable safety profile (16).

In summary, ACC15 expands the horizons of our knowledge and provides new perspectives for trial designs, risk assessment models, current treatment algorithms with novel agents, optimizations of device therapies, percutaneous and surgical treatments in different aspects of cardiovascular medicine

\section{References}

1. Gillinov AM, Gelijns AC, Parides MK, DeRose JJ Jr, Moskowitz AJ, Voisine $\mathrm{P}$, et al. Surgical ablation of atrial fibrillation during mitralvalve surgery. N Engl J Med 2015; 372: 1399-409. [CrossRef]

2. DiBiase D. Ablation vs. Amiodarone for treatment of persistent atrial fibrillation in patients with congestive heart failure and an implanted device: Results from the AATAC Multicenter Randomized Trial. Presented at: American College of Cardiology/i2 Scientific Session; March 16, 2015; San Diego, CA.

3. Mega JL, Walker JR, Ruff CT, Vandell AG, Nordio F, Murphy SA, et al. Genetics and the clinical response to warfarin and edoxaban: findings from the randomised, double-blind ENGAGE AF-TIMI 48 trial. Lancet 2015: pii: S0140-6736(14)61994-2.

4. Pathak RK, Middeldorp ME, Meredith M, Mehta AB, Mahajan R, Wong CX, et al. Long-Term Effect of Goal Directed Weight Management in an Atrial Fibrillation Cohort: A Long-term Follow-Up StudY (LEGACY Study). J Am Coll Cardiol 2015; 65: 2159-69. [CrossRef]

5. Hausenloy DJ. Effect of remote ischemic preconditioning on clinical outcomes in patients undergoing coronary artery bypass graft surgery: a multi-center randomized controlled clinical trial. Presented at: American College of Cardiology/i2 Scientific Session; March 16, 2015; San Diego, CA.

6. Sabatine MS, Giugliano RP, Wiviott SD, Raal FJ, Blom DJ, Robinson J, et al.; Open-Label Study of Long-Term Evaluation against LDL Cholesterol (OSLER) Investigators. Efficacy and safety of evolocumab in reducing lipids and cardiovascular events. $\mathrm{N}$ Engl $\mathrm{J}$ Med 2015; 372: 1500-9. [CrossRef]

7. Robinson JG, Farnier M, Krempf M, Bergeron J, Luc G, Averna M, et al; ODYSSEY LONG TERM Investigators Efficacy and safety of alirocumab in reducing lipids and cardiovascular events. $\mathrm{N}$ Engl $\mathrm{J}$ Med 2015; 372: 1489-99. [CrossRef]

8. Stone NJ, Lloyd-Jones DM. Lowering LDL cholesterol is good, but how and in whom? N Engl J Med 2015; 372: 1564-5. [CrossRef]

9. Reddy M, Atoui M, Pillarisetti J, Gunda S, Atkins D, Bommana S, et al. Externalization of Conductor Cables in QuickSite and QuickFlex Left Ventricular Leads. ACC 15 Moderated Poster presentation No 1129M-03, March 14, 2015 San Diego, CA.

10. Maron BJ, Rowin EJ, Casey S, Link M, Lesser JR, Chan RHM, et al. Paradigm Change in the Demographics of Mortality in Hypertrophic Cardiomyopathy. ACC 15 Moderated Poster presentation no 1269M-07, March 16, 2015 San Diego, CA.

11. Svatikova A, Covassin N, Somers K, Soucek F, Kara T, Ruzek L, et al. Potentiated Blood Pressure Responses to Energy Drink Intake in Caffeine Naïve Healthy Adults: A Double Blind Randomized Controlled Study. ACC 15 Poster presentation no 1141-134, March 14, 2015 San Diego, CA.

12. Durup D, Jørgensen HL, Christensen J, Tjønneland A, Olsen $A$, Halkjær $\mathrm{J}$, et al. A reverse $\mathrm{J}$-shaped association between serum 25-hydroxyvitamin $D$ and cardiovascular disease mortality - the CopD-study. J Clin Endocrinol Metab 2015; 100: 2339-46. [CrossRef]

13. Bergh C, Udumyan R, Fall K, Almroth H, Montgomery S. Stress resilience and physical fitness in adolescence and risk of coronary heart disease in middle age. Heart 2015; 101: 623-9. [CrossRef] 
14. Huo Y, Li J, Oin X, Huang Y, Wang X, Gottesman RF, for the CSPPT Investigators. Efficacy of folic acid therapy in primary prevention of stroke among adults with hypertension in China the CSPPT randomized clinical trial. JAMA 2015; 313: 1325-35. [CrossRef]

15. Franzese CJ, Bliden KP, Gesheff MG, Pandya S, Guyer KE, Singla $A$, et al. Relation of fish oil supplementation to markers of atherothrombotic risk in patients with cardiovascular disease not receiving lipid-lowering therapy. Am J Cardiol 2015; 115: 120411. [CrossRef]

16. McLaughlin VV, Channick R, Chin K, Frey A, Gaine S, Ghofrani A, et al., Oral presentation: Effect of selexipag on morbidity/mortality in pulmonary arterial hypertension: results of the GRIPHON study. Presented at: American College of Cardiology/i2 Scientific Session; Abstract No: 906-12. March 15, 2015, 10:45-12:15pm. San Diego, CA. 